\title{
Numerical study of micromixing combining alternate flow and obstacles ${ }^{2}$
}

\author{
J.M. Miranda ${ }^{\text {a, }}$, H. Oliveira ${ }^{\text {b }}$, J.A. Teixeira ${ }^{c}$, A.A. Vicente ${ }^{c}$, J.H. Correia ${ }^{b}$, G. Minas ${ }^{\text {b }}$ \\ a University of Porto, Engineering Faculty, Rua Dr. Roberto Frias, 4200-465 Porto, Portugal \\ b University of Minho, Biological Engineering Center, Campus de Gualtar, 4710-057 Braga, Portugal

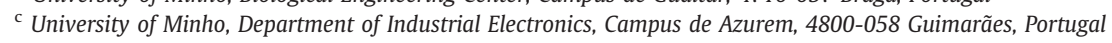

\section{A R T I C L E I N F O}

Available online 16 March 2010

\section{Keywords:}

Alternate flow

Fluidic mixer

Passive mixing

Active mixing

Microfluidics

\begin{abstract}
A B S T R A C T
Mixing in microsystems, combining alternate flow with obstacles, is studied by numerical methods. Simulations show that the layers of high and low solute concentrations, created by the alternate flow, are split into smaller chunks of fluid by obstacles inserted in the mixing channel, decreasing the critical mixing length. Reverse flow is necessary to guarantee symmetry and good mixing. Mixing increases with the increase of the number of obstacles. Increase of frequency improves mixing but requires an increase of reverse flow. The improvement in the mixing process shows that this method is very useful for designing mixers for lab-on-a-chip devices.
\end{abstract}

(c) 2010 Elsevier Ltd. All rights reserved.

\section{Introduction}

The healthcare sector is nowadays one of the most dynamic and where the novelty is a strategic and operational imperative. The possibility to perform clinical analyses with instantaneous results and outside the clinical laboratories has led to the development of microfluidic devices with the fluidic, detection and readout systems integrated in a single-chip [1]. The advantages associated with shrinking clinical analysis systems include: small sample volume, high degree of system integration, automation of measurement, short response time, improved analytical performance, laboratory safety and reduced cost [2]. In clinical analysis laboratories, chemical species are detected by spectrophotometry. Spectrophotometry based on colorimetric detection allows the selective detection of the biomolecules concentration in biological fluids samples [3]. The measurement is based on optical absorption in a part of the visible spectrum defined by the reaction of the specific biomolecule with a specific reagent. In addition, the biomolecule concentration is measured by using a mixture of a reagent with a sample.

An essential requirement for any practical fully integrated lab-on-achip device is the ability to mix two or more fluids thoroughly and efficiently, i.e., in a reasonable amount of time. The microscale conditions have distinctive properties due to its small dimensions and typically low volume flow rate [4]. Rapid mixing becomes a challenging task, as due to strictly laminar flow conditions (it is generally operated at Reynolds numbers of less than 1), turbulent diffusion is absent and the mixing must be achieved by molecular diffusion (which is a rather slow process, even over short distances [5]) or by chaotic advection [6-8]. Therefore, while small molecules can diffuse significant distances during

\footnotetext{
is Communicated by W.J. Minkowycz.

* Corresponding author.

E-mail address: jmiranda@fe.up.pt (J.M. Miranda).
}

the average residence time in the device, large molecules or particles that do not diffuse significantly during the same interval, will not move appreciably from their original stream.

The critical dimension that governs the extent of interdiffusion is the dimension along which diffusion occurs between streams. In a conventional T mixer, this length is equal to half the channel width (supposing the streams have the same flow rate). For a uniaxial flow, the channel length required for mixing is roughly equal to the product of the Peclet number by the width of the channel: $P e \times W$ [6]. This mixing length can be prohibitively long if the flow velocity is too high and the diffusion coefficient of at least one of the components is too small. Since most biological fluid analysis applications require the use of enzymatic reactions, the diffusion coefficient of the enzyme is frequently the limiting factor.

It is possible to improve mixing by increasing the interfacial mass transport area and by decreasing the mixing length. In the literature, two main approaches are followed, passive mixing [6,9-11], which relies on specially conceived static microstructures, usually tridimensional, and active mixing [12-22], which relies on the application of periodic external fields to the flow, achieved by the use of MEMS (Micro Electro Mechanical Systems) devices, such as microvalves or micropumps that increase the cost, need complex control systems and could be difficult to integrate [23].

A common form of active mixing is mixing by using pressure driven periodic flows [12-15,19-22], which will create a flow with layers of low and high solute concentrations along the mixing channel. However, the thickness of these layers should be smaller than the half channel width.

Pressure driving flows have parabolic velocity profiles. The parabolic profile contributes to mixing, since different layers will interpenetrate each other allowing mixing by diffusion in the transversal direction. However, the fluid in the center of the channel has a smaller residence 


\begin{tabular}{|c|c|}
\hline \multicolumn{2}{|c|}{ Nomenclature } \\
\hline$c$ & Normalized concentration \\
\hline$C_{i}$ & $\begin{array}{l}\text { Concentration of each point in the vertical line sampled } \\
\text { several times during a complete cycle }\end{array}$ \\
\hline$\overline{\mathrm{C}}$ & Concentration of a perfectly mixed solution \\
\hline$D$ & Diffusivity \\
\hline$f$ & Frequency \\
\hline$l_{\mathrm{e}}$ & Normalized length of the ejector \\
\hline$L_{\mathrm{e}}$ & Length of the ejector \\
\hline$l_{\mathrm{f}}$ & Normalized length of the feed channels \\
\hline$L_{\mathrm{f}}$ & Length of the feed channels \\
\hline$l_{\mathrm{m}}$ & Normalized length of the mixing channel \\
\hline$L_{\mathrm{m}}$ & Length of the mixing channel \\
\hline$M$ & Mixing index \\
\hline$M_{0}$ & Mixing index in a mixer without obstacles \\
\hline$M_{5}$ & Mixing index in a mixer with 5 obstacles \\
\hline$p$ & Normalized pressure \\
\hline$P_{0}$ & Reference pressure \\
\hline$t$ & Non-dimensional time \\
\hline$T$ & Dimensional time \\
\hline$V_{0}$ & Maximum feed velocity (for $\beta=1.0$ ) \\
\hline$V_{\mathrm{P}}$ & Velocity of the pure solvent stream \\
\hline$V_{\mathrm{T}}$ & Velocity of the stream containing the tracer \\
\hline$V_{\mathrm{x}}$ & Tangential component of the local velocity \\
\hline$\vec{v}$ & Normalized velocity vector \\
\hline$\vec{V}$ & Velocity vector \\
\hline $\bar{V}$ & Average velocity \\
\hline W & Width of the feed channel \\
\hline$w_{\mathrm{e}}$ & Normalized width of the ejector \\
\hline$W_{\mathrm{e}}$ & Width of the ejector \\
\hline$w_{\mathrm{m}}$ & Normalized width of the mixing channel \\
\hline$W_{\mathrm{m}}$ & Width of the mixing channel \\
\hline$w_{\text {obs }}$ & Normalized width of the obstacle \\
\hline$W_{\text {obs }}$ & Width of the obstacle \\
\hline$x$ & Coordinate normalized by $\mathrm{W}$ \\
\hline$y$ & Coordinate normalized by $\mathrm{W}$ \\
\hline$X$ & Coordinate \\
\hline Y & Coordinate \\
\hline \multicolumn{2}{|c|}{ Non-dimensional numbers } \\
\hline$E u_{0}$ & Euler number \\
\hline $\mathrm{Pe}$ & Peclet number \\
\hline $\operatorname{Re}$ & Reynolds number \\
\hline St & Strouhal number \\
\hline Sc & Schmidt number \\
\hline \multicolumn{2}{|c|}{ Greek symbols } \\
\hline$\beta$ & Constant \\
\hline$\phi_{\mathrm{T}}$ & Initial phase of the stream containing the tracer \\
\hline$\phi_{\mathrm{P}}$ & Initial phase of the pure solvent stream \\
\hline$\mu$ & Viscosity \\
\hline$\rho$ & Density \\
\hline
\end{tabular}

time and a high local Peclet number. In continuous flow, good mixing can be obtained by splitting the fluid [9]. However, this technique is only efficient if it is possible to rotate the fluid tridimensionally. Splitting without rotation is inefficient.

Active mixing using periodic flow can be improved if combined with passive mixing [24]. In this work, the combination is made by using alternate flow in a mixer with obstacles. The function of the alternate flow is to create alternate layers of low and high concentrations. The function of the obstacles is to delay the fluid in the center of the mixing channel and to split the layers into smaller chunks that are easily mixable by diffusion.

\section{Mixer description}

Preliminary work was done to optimize the mixer. The mixer was designed so that the layers created by the alternate flow must be symmetrical and thinner than half the width of the mixing channel. A conventional $\mathrm{T}$ mixer is not adequate because the smaller layers of flow are not symmetrical and larger layers which are symmetrical are too large. For this reason, the conventional $\mathrm{T}$ mixer was modified. It was introduced an ejector, which has a width smaller than the width of the mixing channel (Fig. 1). The mixer has two entries, one for each reacting stream. The purpose is to mix the reacting streams.

Four versions of the mixer were studied, a version without an obstacle (Fig. 1a), a version with a central obstacle (Fig. 1b), a version with three obstacles (Fig. 1c) and a version with five obstacles (Fig. 1d). All versions have two feed channels (of width $W$ and length $L_{\mathrm{f}}$ ) and an ejector (of width $W_{\mathrm{e}}$ and length $L_{\mathrm{e}}$ ), which connects to the mixing channel (of width $W_{\mathrm{m}}$ and length $L_{\mathrm{m}}$ ). The width of the squared obstacle is $W_{\text {obs. }}$. The width of the feed channel, $W$, was taken as the characteristic dimension. The following non-dimensional geometrical variables were defined:

$$
\begin{aligned}
& l_{\mathrm{f}}=L_{\mathrm{f}} / W ; l_{\mathrm{e}}=L_{\mathrm{e}} / W ; l_{\mathrm{m}}=L_{\mathrm{m}} / W \\
& w_{\mathrm{e}}=W_{\mathrm{e}} / W ; w_{\mathrm{m}}=W_{\mathrm{m}} / W ; w_{\mathrm{obs}}=W_{\mathrm{obs}} / W
\end{aligned}
$$

\section{Simulations}

Numerical methods are used to simulate the alternate flow and the mixing of a passive tracer (Fig. 2). One of the streams is a pure solvent stream, like water, and the other is a solvent stream containing a passive tracer.

Alternate flow (Fig. 3) is described by the following equation:

$$
\left\{\begin{array}{l}
V_{\mathrm{T}}=0.5 V_{0}\left\{1+\beta \operatorname{sgn}\left[\sin \left(2 \pi S t \times t+\phi_{\mathrm{T}}\right)\right]\right\} \\
V_{\mathrm{P}}=0.5 V_{0}\left\{1+\beta \operatorname{sgn}\left[\sin \left(2 \pi S t \times t+\phi_{\mathrm{P}}\right)\right]\right\}
\end{array}\right.
$$

where $\operatorname{sgn}()$ is the sign function

$$
\operatorname{sgn}(x)=\left\{\begin{aligned}
-1 & \text { for } x<0 \\
0 & \text { for } x=0 \\
1 & \text { for } x>0
\end{aligned}\right.
$$

$V_{0}$ is the mean feed velocity, St is the Strouhal number based on the width of the channel $(W), \phi_{\mathrm{P}}$ is the initial phase of the pure solvent

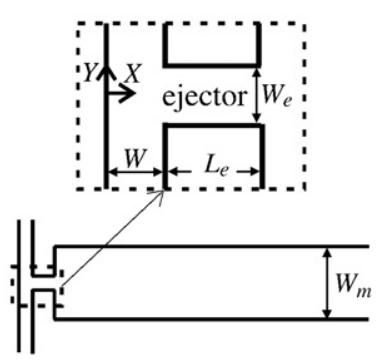

b

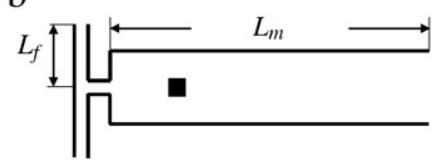

$\mathrm{C}$

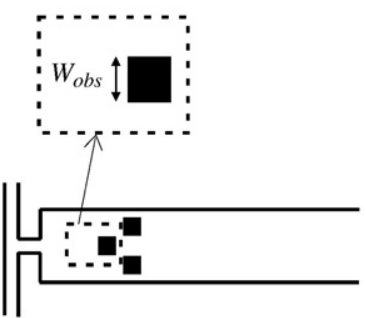

$\mathrm{d}$

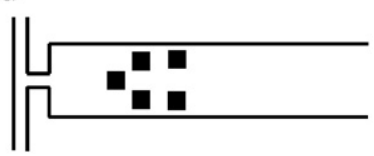

Fig. 1. Schematic representation of the mixers studied. 


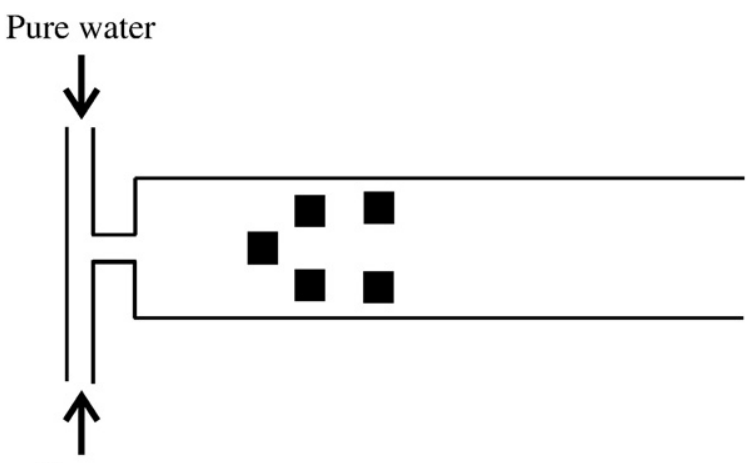

Tracer

Fig. 2. Mixing of a stream containing a passive tracer with a stream of pure water.

stream, $\phi_{\mathrm{T}}$ the initial phase of the stream containing the tracer and $\beta$ is a constant. If this constant is higher than 1 the velocities are negative during half of the cycle and above $V_{0}$ in the other half of the cycle.

The non-dimensional time, $t$, is given by:

$t=V_{0} T / W$

where $T$ is the dimensional time. The Strouhal number is given by:

$S t=f W / V_{0}$

where $f$ is the frequency of the alternate flow.

The mixing of a passive tracer was simulated using the commercial computational fluid dynamics software, Fluent ${ }^{\mathrm{TM}}$. The grids (Fig. 4) were generated by GAMBITTM . The grids are uniform and the grid spacing is equal to $0.05 \times \mathrm{W}$.

Fluent ${ }^{\mathrm{TM}}$ solves the Navier-Stokes equations and the mass transport equations for each component. The Navier-Stokes equations are:

$\frac{\partial \vec{v}}{\partial t}+(\vec{v} \cdot \nabla) \vec{v}=-E u_{0} \nabla p+\frac{1}{R e} \nabla^{2} \vec{v}$

$E u_{0} \Delta p=-\nabla v_{x} \frac{\partial \vec{v}}{\partial x}-\nabla v_{y} \frac{\partial \vec{v}}{\partial y}-\nabla v_{z} \frac{\partial \vec{v}}{\partial z}$

where $x, y$ and $z$ are the coordinates normalized by $W$ and $E u_{0}$ is the Euler number:

$E u_{0}=P_{0} / \rho V_{0}$

and $R e$ is the Reynolds number based on the channel width:

$\operatorname{Re}=\rho V_{0} W / \mu$

Velocity is given by Eq. (3). Along the wall of the mixer and at the surface of obstacles, the no-slip condition was considered. $\mathrm{a}$
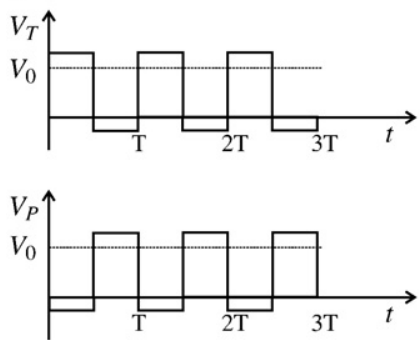

b
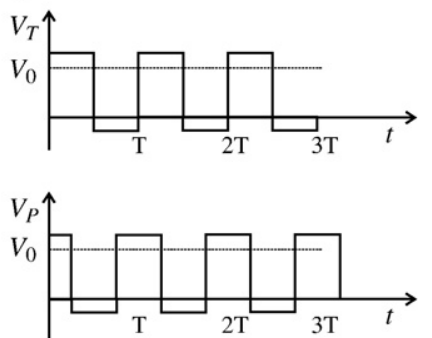

a

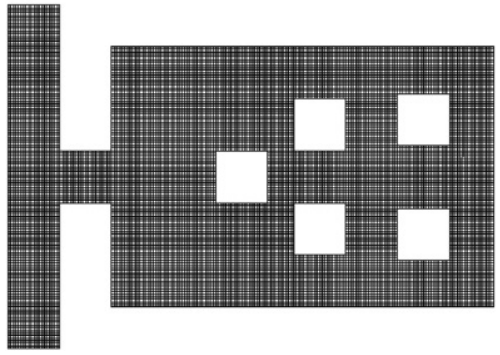

Fig. 4. a) Grid for the five obstacles version; b) detail of the ejector. b

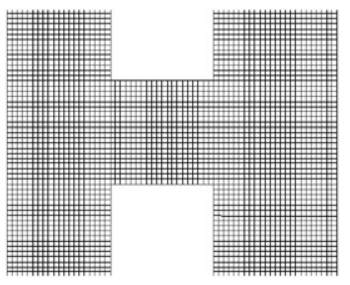

The mass transport equation for the passive tracer is:

$\frac{\partial c}{\partial t}+\vec{v} \cdot \nabla c=\frac{1}{P e} \Delta c$

$P e$ is the Peclet number defined by:

$P e=V_{0} W / D$

where $D$ is the diffusivity of the passive tracer.

The time dependent Eqs. (6) and (10) were solved by a first order implicit unsteady method. The SIMPLE algorithm was used to solve the flow equations. The convection term of the flow equations was approximated by a first order upwind method. The mass transport equations were solved by a second order upwind method. The periodic inlet boundary conditions were handled by using 30 time steps per cycle with a maximum of 20 iterations per time step.

Concentration of the tracer is equal to 1 in the upper feed channel (Fig. 2) and equal to zero in the lower feed channel. A zero mass flux is considered along the walls and a zero diffusive flux is considered at the exit of the mixer.

Mixing was measured in vertical lines along the mixing channel. Mixing was quantified by (see $[13,14])$ :

$M=1-\sqrt{\frac{\sum_{i=1}^{N}\left(\frac{c_{i}}{\overline{\bar{C}}}-1\right)^{2}}{N}\left|\frac{V x_{i}}{\bar{V}}\right|}$

where $C_{\mathrm{i}}$ is the concentration of each point in the vertical line sampled several times during a complete cycle, $V x_{\mathrm{i}}$ is the tangential component of the local velocity, $\bar{C}$ is the concentration of a perfectly mixed solution and $\bar{V}$ is the average velocity.

\section{Dimensional analysis}

To simplify the study, dimensional analysis was used to identify the smallest set of non-dimensional numbers that influence mixing in the device. Since mixing depends on the local velocity of the fluid, the analysis starts by finding the non-dimensional numbers that influence the local velocity.

The local velocity $\mathrm{V}$ in a given position of the mixer is a function of the coordinates of the point, $X$ and $Y$, the time $T$, the width of the channel $W$, the feed velocity $\mathrm{V} 0$, the frequency of the alternate flow $\mathrm{f}$, the constant $\beta$, the initial phase of each stream, the fluid transport properties (viscosity, $\mu$, and density, $\rho$ ) and the geometry of the mixer $(G)$ :

$V=f_{1}\left(X, Y, T, W, V_{0}, f, \beta, \phi_{R}, \phi_{S}, \mu, \rho, G\right)$

The number of parameters of this relation can be significantly reduced by dimensional analysis:

$v=f_{2}\left(x, y, t, \beta, \phi_{\mathrm{T}}-\phi_{\mathrm{P}}, \operatorname{Re}, S t, \mathrm{G}\right)$

Fig. 3. Inflow boundary conditions: a) $\phi_{\mathrm{T}}-\phi_{\mathrm{P}}=\pi$; (b) $\phi_{\mathrm{T}}-\phi_{\mathrm{P}}=\pi / 2$. 
a

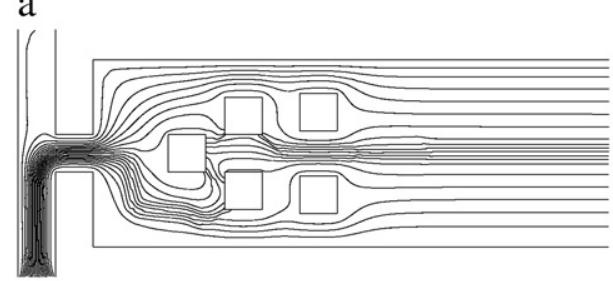

b

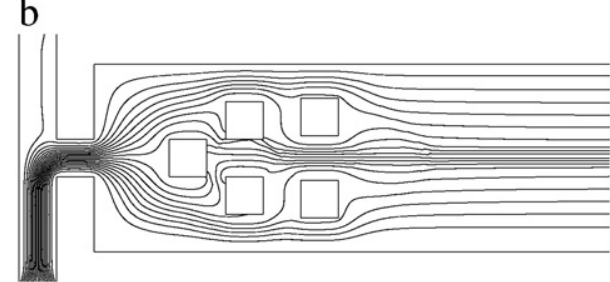

c

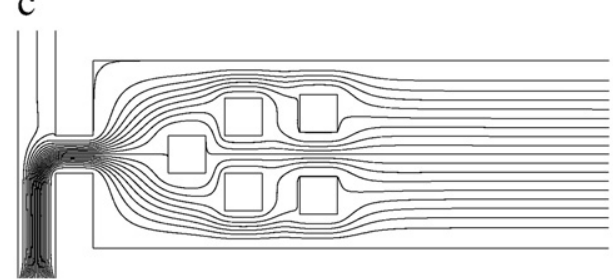

d

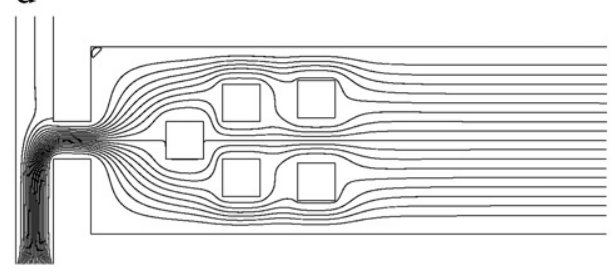

$\mathrm{e}$

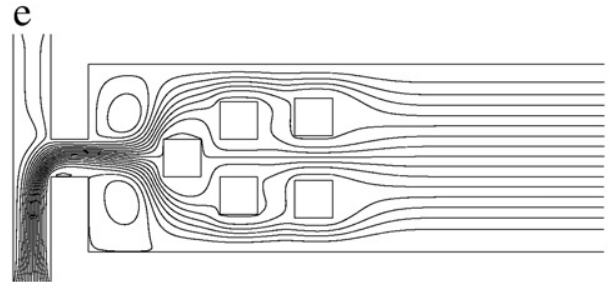

f

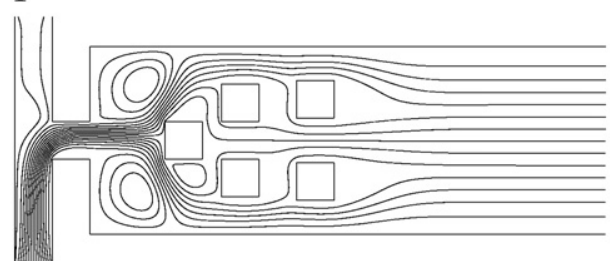

Fig. 5. Stream lines for $V_{\mathrm{T}}=0.0011 \mathrm{~m} / \mathrm{s}, V_{\mathrm{P}}=0.0001 \mathrm{~m} / \mathrm{s}, \beta=1.1$ and different Reynolds numbers: a) $\operatorname{Re}=0.01$; b) $\operatorname{Re}=0.1$; $) \operatorname{Re}=1$; d) $\operatorname{Re}=10$; e) $\operatorname{Re}=50$; f) $\operatorname{Re}=100$.

The local concentration, $C$, is influenced by the flow field and will depend on the same variables as the local velocity. Additionally, the local concentration is a function of the Schmidt number and of the feed concentration.

$c=g\left(x, y, t, \beta, \phi_{\mathrm{T}}-\phi_{\mathrm{P}}, R e, S c, S t, \mathrm{G}\right)$

Since mixing is independent of $Y$, then

$M=h\left(x, t, \beta, \phi_{\mathrm{T}}-\phi_{\mathrm{P}}, \operatorname{Re}, \operatorname{Re}, S c, S t, \mathrm{G}\right)$

For small Reynolds numbers, as the ones in microfluidic systems, the flow pattern is independent of the Reynolds number. In this case, the Schmidt and the Reynolds numbers can be grouped. The functional dependence of the mixing parameter becomes:

$M=h_{2}\left(x, t, \beta, \phi_{\mathrm{T}}-\phi_{\mathrm{P}} P e, S t, \mathrm{G}\right)$

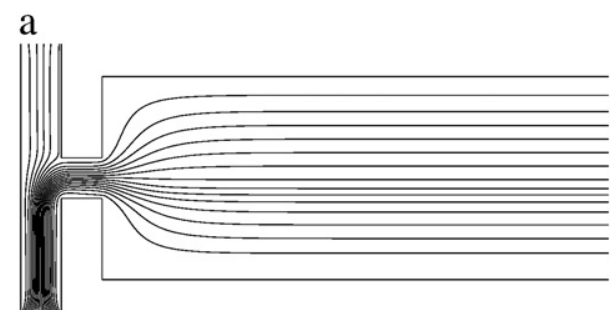

b

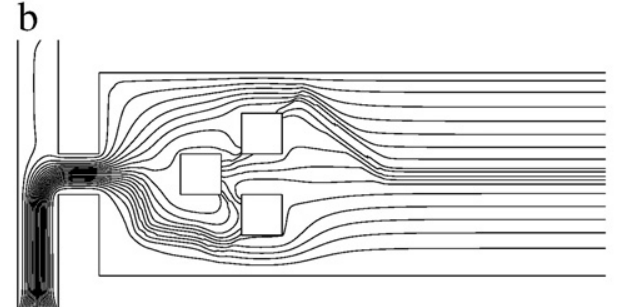

In the steady state, mixing is independent of $t$ and a function of the position along the mixing channel, the phase difference, the Peclet and Strouhal numbers and the constant $\beta$.

$M=h_{2}\left(x, \beta, \phi_{\mathrm{T}}-\phi_{\mathrm{P}} P e, S t, \mathrm{G}\right)$

\section{Results}

Flow and concentration fields were determined for the four versions of the mixers (see Fig. 1) and for three operating conditions:

a) Continuous flow;

b) Alternate flow with $\phi_{\mathrm{T}}-\phi_{\mathrm{P}}=\pi$;

c) Alternate flow with $\phi_{\mathrm{T}}-\phi_{\mathrm{P}}=\pi / 2$;

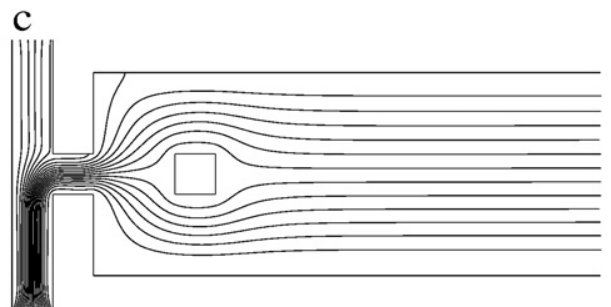

d

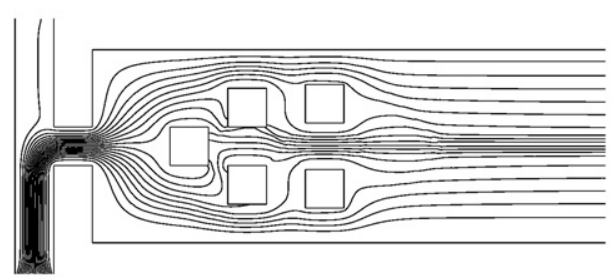

Fig. 6. Stream lines for $\operatorname{Re}=0.1, V_{\mathrm{T}}=0.0011 \mathrm{~m} / \mathrm{s}, V_{\mathrm{P}}=0.0001 \mathrm{~m} / \mathrm{s}, \beta=1.1$ and different geometries. 

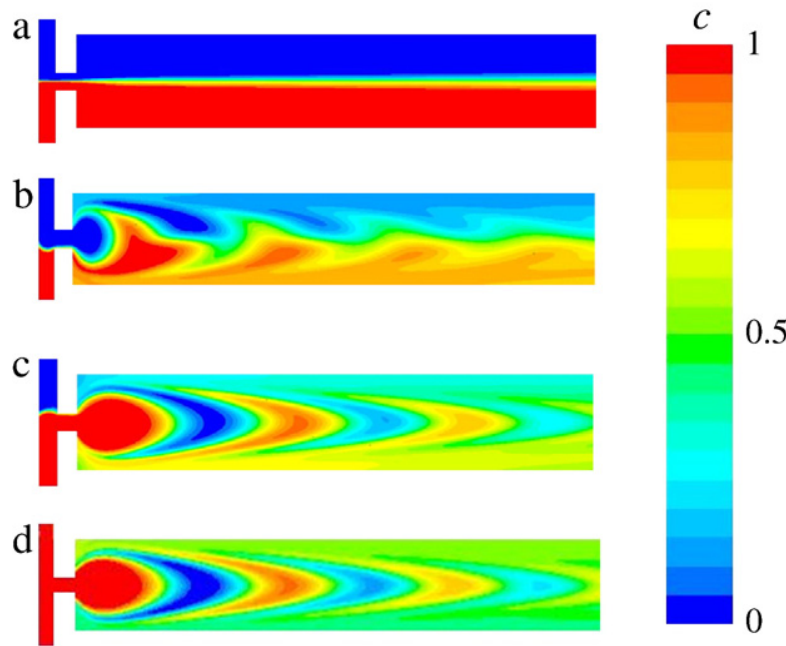

Fig. 7. Concentration field for steady state $\left(R e=0.1, P e=1450, S t=3.33 \times 10^{-2}, w_{o b s}=1\right.$, $w_{\mathrm{e}}=1$ and $w_{m}=5$ ): (a) continuous flow; (b) alternate flow with $\phi_{\mathrm{T}}-\phi_{\mathrm{P}}=\pi / 2$ and $\beta=1.0$; (c) alternate flow with $\phi_{\mathrm{T}}-\phi_{\mathrm{P}}=\pi$ and $\beta=1.0$; (d) alternate flow with an obstacle and $\phi_{\mathrm{T}}-\phi_{\mathrm{P}}=\pi$ and $\beta=4$.

\subsection{Flow fields}

For each case, the steady state flow field is a function of the Reynolds number (Fig. 5), the Strouhal number, the reverse flow parameter ( $\beta$ ) and of the geometry of the mixer (number of obstacles, Fig. 6). Numerical results show that the flow is independent of the Reynolds number for Reynolds numbers less than 1 . For Reynolds number higher than 1 the inertial effects start to become important. However, the Reynolds number is very low in microfluidic devices, often bellow 1 .

\subsection{Concentration fields}

Mass transport results are presented in Fig. 7. Mixing for continuous flow (Fig. 7a) is very small when compared with mixing for alternate flow (Fig. $7 \mathrm{~b}$ to $\mathrm{d}$ ).

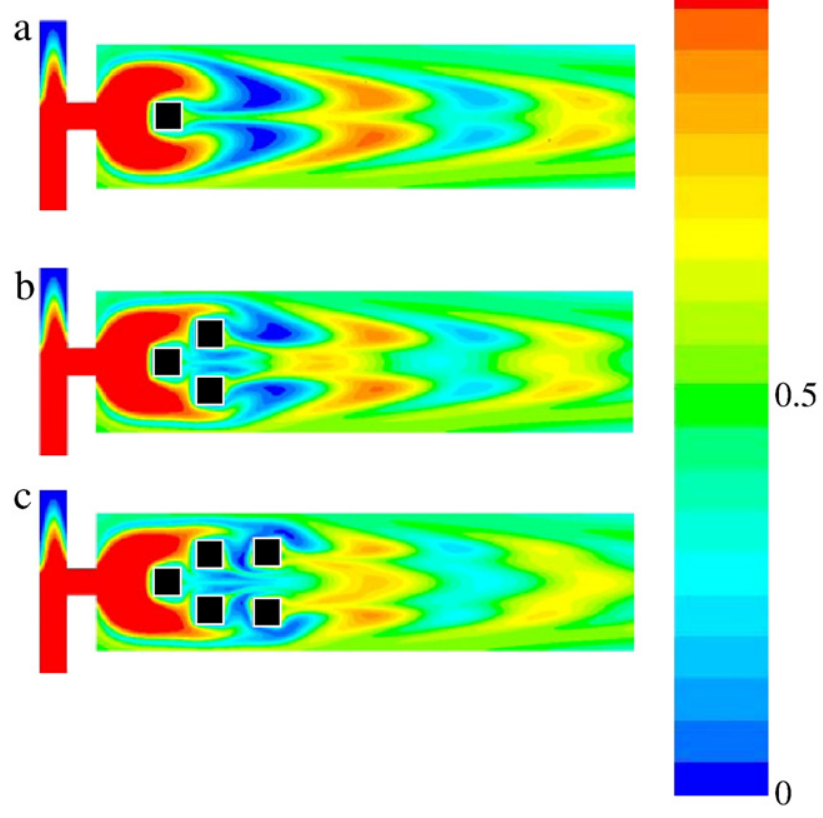

Fig. 8. Concentration field for steady state $\left(R e=0.1, P e=1450, S t=3.33 \times 10^{-2}, \phi_{\mathrm{T}}-\phi_{\mathrm{P}}=\pi\right.$, $w_{o b s}=1, w_{\mathrm{e}}=1, \beta=1.1$ and $w_{\mathrm{m}}=5$ ): (a) alternate flow with an obstacle; (b) alternate flow with three obstacles; (c) alternate flow with five obstacles.

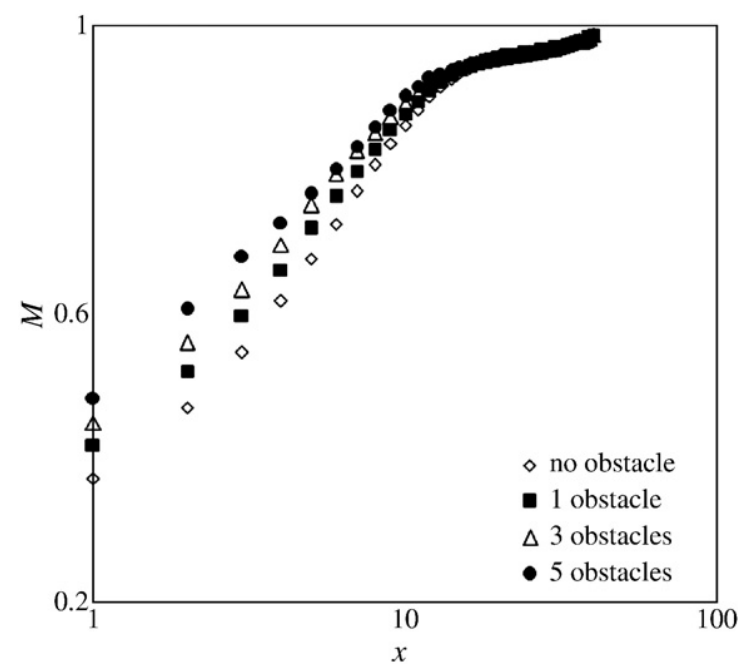

Fig. 9. Mixing index along the mixing channel ( $R e=0.1, P e=1450, S t=3.33 \times 10^{-2}$, $w_{o b s}=1, w_{\mathrm{e}}=1 . \phi_{\mathrm{T}}-\phi_{\mathrm{P}}=\pi \cdot \beta=1.2$, and $\left.w_{\mathrm{m}}=5\right)$.

The mixer geometry facilitates the creation of layers of fluid of different compositions (Fig. 7c and d). Due to the parabolic flow, the layers have a curved interface between each other. Mixing is improved due to the increase of the area between different layers and Taylor dispersion. However, the fluid in the center of the channel doesn't mix as effectively as the fluid near the wall because the residence time and the thickness of the layer are larger.

Comparison between Fig. $7 \mathrm{~b}$ and $\mathrm{c}$ shows that the mixing is more efficient for the phase difference equal to $\pi$. The concentration field in Fig. $7 \mathrm{~b}$ and $\mathrm{c}$ is asymmetric because the ejector is not completely cleaned after each cycle. This problem can be partially solved by allowing negative inflow velocities $(\beta>1.0)$. Comparison between Fig. $7 c$ and $d$ shows that mixing improves for $\beta>1.0$. The effect of the $\beta$ parameter is related to the Strouhal number. As the Strouhal number increases, the value of $\beta$ required to assure a symmetrical concentration field also increases.

\subsection{Obstacles}

The introduction of an obstacle in the axis of the channel (Fig. 8a) contributes to delay the fluid, to break the layers into smaller chunks and
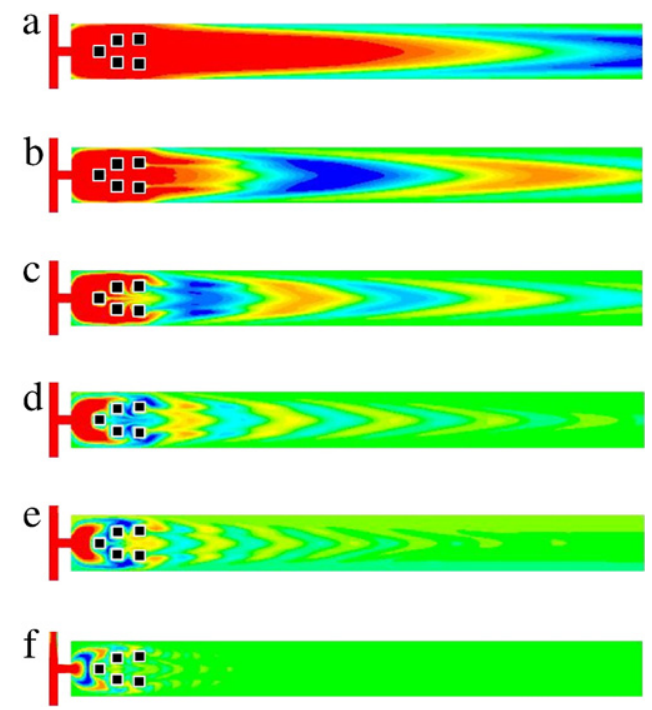

Fig. 10. Concentration fields for steady state $\left(R e=0.1, P e=1450, \phi_{\mathrm{T}}-\phi_{\mathrm{P}}=\pi, w_{\text {obs }}=1\right.$, $w_{\mathrm{e}}=1, l_{\mathrm{e}}=2, \beta=4.0$ and $\left.w_{\mathrm{m}}=5\right):$ (a) $S t=3.33 \times 10^{-3}$; (b) $S t=8.33 \times 10^{-3}$; (c) $S t=1.67 \times 10^{-2}$; (d) $S t=0.0333$; (e) $S t=0.05$; (f) $S t=0.133$. 


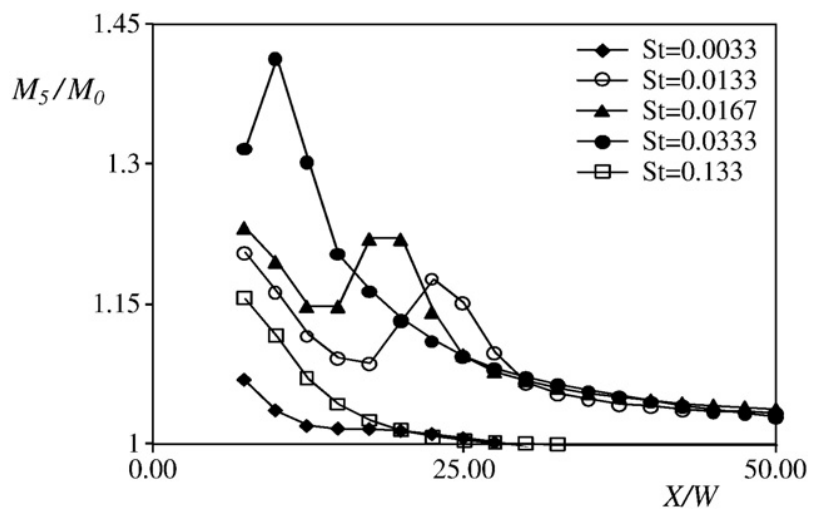

Fig. 11. Ratio between the mixing index with and without obstacles ( $R e=0.1$, $P e=1450, w_{o b s}=1, w_{\mathrm{e}}=1, l_{\mathrm{e}}=2, \phi_{\mathrm{T}}-\phi_{\mathrm{P}}=\pi, \beta=4.0$, and $\left.w_{\mathrm{m}}=5\right)$.

to increase the interfacial area between fluids with different compositions. This effect is further increased by the introduction of more obstacles which break the smaller chunks into smaller ones (Fig. 8b and c).

Fig. 9 shows the mixing index along the axis of the mixing channels for several configurations. The mixing index increases along the axis of the mixing channel and converges to 1 for large values of the distance to the channel entry. With the introduction of obstacles, the mixing index increases in the region located downstream of the obstacles.

\subsection{Influence of the Strouhal number}

Mixing in the device depends on the Strouhal number. Fig. 10 shows the results obtained for Strouhal numbers from $3.33 \times 10^{-3}$ to 0.133 . For $W=10^{-4} \mathrm{~m}$ and $V_{\mathrm{R}}=V_{\mathrm{S}}=10^{-3} \mathrm{~ms}^{-1}$, the Strouhal number of $3.33 \times 10^{-3}$ corresponds to a period of $30 \mathrm{~s}$ and the Strouhal number of 0.133 corresponds to a period of $0.75 \mathrm{~s}$. As the Strouhal number increases, the mixing improves. Increasing the frequency contributes to the decrease of homogeneous chunks of fluid.

The effects of the combination between Strouhal number and obstacles were studied by measuring the ratio between the mixing index with and without obstacles $\left(M_{5} / M_{0}\right) . M_{5}$ is the mixing index with 5 obstacles and $M_{0}$ is the mixing index with no obstacle. Fig. 11 represents the ratio $M_{5} / M_{0}$ along the mixing channel. The results show that obstacles increase mixing. The effect is higher for intermediate Strouhal numbers. In general, the effect of the obstacles decreases along the mixing channel, but the results for intermediate Strouhal numbers have a local maximum.

\section{Conclusion}

Mixing of two streams in a microfluidic system combining alternate flow with obstacles located along the mixing channel was studied by numerical methods. Results show that this method can improve mixing in a T mixer. In addition, for biological fluids analysis using lab-on-a-chip devices, this improvement can lead to shorter mixing channels and to low cost mixers fabricated by planar lithographic technology.

\section{Acknowledgments}

Support for this research was provided by the Engineering School of University of Minho (program IN2TEC) and the Portuguese Science Foundation (grant PTDC/BIO/70017/2006 and grant SFRH/BPD/17689/ 2004).

\section{References}

[1] P. Connolly, Biosensors \& Bioelectronics 10 (1995) 1-6.

[2] S.C. Jakeway, A.J. de Mello, E. Russell, Fres. J. Anal. Chem. 266 (2000) 525-539.

[3] Biochemistry and Organic Reagents: for bioscience investigation. Sigma-Aldrich Diagnostics $\circledR^{2}, 2002$.

[4] S.C. Jakeway, A.J. de Mello, E. Russell, Fres. J. Anal. Chem. 266 (2000) 525-539.

[5] P.C. Simpson, et al., Proc. Natl. Acad. Sci. USA 95 (1998) 2256-2261.

[6] A.D. Stroock, S.K.W. Dertinger, A. Ajdari, I. Mezic, H.A. Stone, G.M. Whitesides, Chaotic mixer for microchannels, Science 295 (2002) 647-651.

[7] Mark A. Stremler, F.R. Haselton, Hassan Aref, Designing for chaos: applications of chaotic advection at the microscale, Phil. Trans. R. Soc. Lond. A 362 (2004) 1019-1036.

[8] X. Niu, Y.K. Lee, Efficient spatial temporal chaotic mixing in microchannels, J. Micromech. Microeng. 13 (2003) 454-462.

[9] Dong Sung Kim, Se Hwan Lee, Tai Hun Kwon, Chong H. Ahn, A serpentine laminating micromixer combining splitting/recombination and advection, Lab Chip 5 (2005) 739-747.

[10] Jessica Melin, Guillem Giménez, Niclas Roxhed, Wouter van der Wijngaart, Göran Stemme, A fast passive and planar liquid sample micromixer, Lab Chip 4 (2004) 214-219.

[11] V. Mengeaud, J. Josserand, H.H. Girault, Mixing processes in a zigzag microchannel: finite element simulations and optical study, Anal. Chem. 74 (2002) 4279-4286.

[12] Tamio Fujiwara, Hiroshi Ohue, Tatsuo Ushijima, Osami Kitoh, Characteristics of Flow Pattern in Alternate Pumping Microreactor, Second International Conference on Transport Phenomena in Micro and Nanodevice, 11-15 June 2006, Barga, Italy.

[13] Arnaud Goullet, Ian Glasgow, Nadine Aubry, Dynamics of microfluidic mixing using time pulsing, Discrete and Continuous dynamical systems, Supplement Volume (2005) 327-336.

[14] Ian Glasgow, Nadine Aubry, Enhancement of microfluidic mixing using time pulsing, Lab Chip 3 (2003) 114-120.

[15] Tamio Fujiwara, Hiroshi Ohue, Tatsuo Ushijima, Osami Kitoh, Optimization of channel geometry and pumping conditions for an alternate pumping microreactor, J. Fluid Sci. Technol. 2 (2007) 389-399.

[16] Tae Gon Kang, Martien A. Hulsen, Patrick D. Anderson, Jaap M.J. den Toonder, Han E.H. Meijer, Chaotic advection using passive and externally actuated particles in a serpentine channel flow, Chem. Eng. Sci. 62 (2007) 6677-6686.

[17] I. Glasgow, J. Batton, N. Aubry, Electroosmotic mixing in microchannels, Lab Chip 4 (2004) 558-562.

[18] Ian Glasgow, Nadine Aubry, Pulsed flow mixing for BIOMEMs applications, 7th international Conference on Miniaturized Chemical and Biochemical Analysts Systems, October 5-9, 2003, Squaw Valley, California USA.

[19] P. Tabeling, M. Chabert, A. Dodge, C. Jullien, F. Okkels, Chaotic mixing in crosschannel micromixers, Phil. Trans. R. Soc. Lond. A 362 (2004) 987-1000.

[20] Kohei Motoo, Naoya Toda, Fumihito Arai, Toshio Fukuda, Kosuke Sekiyama, Masahiro Nakajima, Generation of concentration gradient from a wave-like pattern by high frequency vibration of liquid-liquid interface, Biomed. Microdevices 10 (2008) 329-335.

[21] Xize Niu and Yi-Kuen Lee, Efficient spatial-temporal chaotic mixing in microchannels, J. Micromech. Microeng. 13 (2003) 454-462.

[22] F. Okkels, P. Tabeling, Spatiotemporal resonances in mixing of open viscous fluids, Phys. Rev. Lett. 92 (2004) 038301.1-038301.4.

[23] D.R. Reyes, D. Iossifidis, A. Manz, Micro total analysis systems. 1. Introduction, theory, and technology, Anal. Chem. 74 (2002) 2623-2636.

[24] Cha'o-Kuang Chen, Ching-Chang Cho, A combined active/passive scheme for enhancing the mixing efficiency of microfluidic devices, Chem. Eng. Sci. 63 (2008) 3081-3087. 